\section{Regenerating of Intervertebral Disc}

\section{Shawn P Williams*}

Department of Health Professions, York College, City University of New York, New York, USA

\begin{abstract}
\section{Objective}

To review the existing literature documenting current traditional therapeutic practices being used to treat degenerative disc conditions, and to provide a theoretical framework supporting rehydration capabilities of an Intervertebral Disc (IVD).

\section{Data collection}

Publications produced by various orthopedic-based peer-reviewed journals and spinal decompression companies and were analyzed and compared using conceptual labeling and memos for category building.

\section{Conclusion}

The current practices being used to treat degenerative IVD disorders prove to be both costly and therapeutically ineffective. The data reviewed lend support to the contention that degenerative IVD have the ability to repair, regenerate and rehydrate. Applications of negative-pressure decompressive forces, grant measurable health benefits to patient suffering with condition associated with IVD degeneration. It is plausible that if this area of research is further investigated, then degenerative conditions once believed-by patients and practitioners'-to be permanent may actually be reversed.

Keywords: Rehydrate; Spinal decompression
\end{abstract}

\section{Introduction}

Until recently, physicians specializing in pain management believed that once a significant degenerative spinal condition exists-patients must live with their symptoms or undergo surgical/invasive interventions [1]. However, increasing evidence [2-6] illustrates the ability of degenerate Intervertebral Disc (IVD) disorder (s) to regenerate. This regeneration phenomenon specifically refers to the notion of rehydration. Currently, there is a lack of quality literature that supports the incidence of such findings; however, if this phenomenon

*Corresponding author: KShawn P Williams, Department of Health Professions, York College, City University of New York, New York, USA, Tel: +1 7182622907; E-mail: swilliams2@york.cuny.edu

Citation: Williams SP (2017) Regenerating of Intervertebral Disc. J Phys Med Rehabil Disabil 3: 022.

Received: August 24, 2017; Accepted: September 23, 2017; Published: October 06, 2017 exists, then degenerative IVD conditions once believed by patients and pain management physicians to be permanent may actually be reversed [1]. This paper will attempt to explain how the current literature supports this particular phenomenon by providing a conceptual framework that outlines anatomical, physiological and pathological properties of the intervertebral disc in reference to rehydration capabilities, and reviewing the literature that documents the current traditional therapeutic practices being used to treat degenerative disc conditions. After reviewing these necessary avenues, it should be apparent that the current practices being used to treat degenerative IVD disorders prove to be both costly and therapeutically ineffective. It should also become apparent that based on sound anatomical principles, non-surgical spinal decompression may be able to regenerate/ rehydrate a group of selective degenerative spinal conditions, and therefore can be used as a valuable treatment modality.

\section{Methods}

A search using both the indexed (PubMed and PsychLit) and non-indexed literature was used. Other resources included the Cochrane Library, articles from governing bodies of the Complementary and Alternative Medicine (CAM) profession, trade magazines, and research conferences and symposium proceedings. Conceptual labeling, memos for category building and narrative synthesizing the findings of literature retrieved from searches of computerized databases, hand searches, and authoritative texts.

\section{Discussion}

\section{Anatomical, physiological and pathological properties of the IVD}

The IVD is composed of a nucleus pulposus and annulus fibrosus and is enclosed both above and below by two cartilaginous vertebral endplates. The annulus fibrosus is made up of several lamellae consisting of parallel collagen fibers interdispersed by elastin fibers [3]. Individual lamellae of the annulus fibrosus run obliquely between vertebral bodies, inserting onto the surfaces of the vertebral endplate. This oblique and overlapping arrangement allows a small degree of movement and provides firm stability. However, the posterior aspect of the annulus fibrosus is thinner than the rest of the annulus, which may subject this region to structural weakness; and thus may provide the anatomical basis of more posterior annular tears $[7,8]$.

The nucleus pulposus, located in the center of the disc, consists of a proteoglycan and water matix held together by a network of collagen and elastin fibers [2,9]. The major proteoglycan of the disc is aggrecan [10], which provides the nucleus' ability to attract and retain water. Initially, an estimated 70 to $90 \%$ of the content of the nucleus is water [8], which is typically at its greatest capacity in the young adult, and then declines thereafter [9]. By a series of complex interactions, the collagen-proteoglycan-water complex provides the osmotic properties that enable the intervertebral disc to resist spinal compressive forces, while permitting limited ranges of motion $[9,10]$. Thus, the water content of the nucleus pulposus may be a reliable measure of its degenerative status. 
The outmost layer of the annulus fibrous is the only portion of the entire intervertebral disc complex that receives blood supply; and in fact, the healthy IVD is mostly avascular $[9,10]$. Because of the lack of blood vessels within the nucleus, the nucleus pulposus obtains its nutrition by a combination of diffusion and fluid flow [11] via the adjacent blood vessels and possibly via a mechanical phenomenon referred to as imbibition and compressive loading coupling [1,8]. Repetitive traction followed by compression of the disc produce "imbibition and osmosis" of water into the disc, rehydrating or regenerating the disc height [1].

Because the IVD undergoes very extensive destructive changes with age and degeneration throughout life [10], the exact process of disc degeneration is somewhat inconsistent. Adams and Roughley [9] published a review documenting the diversity of disc degeneration definitions, with the objective of distinguishing IVD degeneration from physiologic processes of aging, healing, and remodeling. As a result, the authors define the disc degeneration process as "an $a b$ errant, cell-mediated response to progressive structural failure" [9]. They also state that a degenerative disc is "one with structural failure combined with accelerated or advanced signs of aging" [9].

An accurate definition may aid in better serving patients, in terms of prognosis, optimal therapeutic interventions, and identifying risk factors and prevention strategies. Thus, in order to better understand healing possibilities and/or capabilities, it may be necessary to understand the nature of degeneration. As such, the majority of the scientific literature suggests that IVD degeneration increases with age. Environmental risk factors, genetic precursors, and nutritional effects appear to accelerate the aging and degenerative processes. There are some reports $[9,11]$ that suggest genetic inheritance is the highest risk factor; however Adams et al., [9] report that these accusations may not be credible due to inevitable environmental influences. Nonetheless, it is agreed upon within the literature that genetic inheritance can alter specific genes, which can ultimately affect the strength and stability of the important holding elements, such as collegan, aggrecan, vitamin D, and cartilage proteins. Environmental risk factors for disc degeneration include high and repetitive mechanical loading and smoking cigarettes $[8,9]$. Repetitive mechanical loading refers to combined loading, bending and compression performed at a high frequency, beyond physiological limits. Excessive mechanical loading and/or vibration forces disrupt a disc structure and imitate a cascade of cell-mediated responses, leading to further disruption. Other influences that contribute to a weakening of the disc, and subsequently structure failure, include age, impaired diffusion, altered levels of cellular enzyme activity, structural failure, and neurovascular growth.

Although the etiology and epidemiology of disc degeneration is far from being fully understood, there is a consensus that multiple events contribute to the complex phenomena of disc degeneration. The processes of aging and injury-related degeneration can alter diffusion efficiency; therefore, IVDs have only a limited ability to recover from any metabolic or mechanical injury [9]. However, because of a rather improved understanding of the cellular and molecular events of disc aging and degeneration, newer concept (s) of biologic disc repair is constantly being developed.

Regardless of the cause of injury, a gradual loss of proteoglycans and water initiates the degenerative process [12-14]. One report suggests that a water loss of only 12 percent will reduce the disc height by 50 percent; resulting in a loss of disc volume [1]. As mentioned-water content of the nucleus pulposus can be a reliable measure of its degenerative status. Degeneration status can be visualized and measured using specific Magnetic Resonance Imaging (MRI) signals [2]. If a specific therapy is applied to a degenerative disc, then regeneration and/or rehydration possibilities may be identified with pre-and-post MRI studies. Performing pre-and-post MRI's, in conjunction with non-surgical spinal decompression therapy, may prove that the above rehydration phenomenon can exist. If rehydration can if fact occur on a selected group of degenerative IVD conditions, then these conditions should be appropriately identified. Differential diagnosis serves as a screening tool for prospective patients that may benefit from non-surgical spinal decompression.

\section{Differential diagnosis}

An accurate definition of disc degeneration should ultimately provide the ability to generate an accurate clinical diagnosis. In turn, this precision may aid in better serving patients, in terms of prognosis, optimal therapeutic interventions, and identifying risk factors and prevention strategies.

Crock [15] identified the term internal Disc Disruption (IDD), and described it as a condition marked by an alteration in the internal structure and metabolic functions of the IVD, usually preceded by injuries. Injuries that affect the inner annulus or endplate decompress the nucleus, and healing processes are often overtaken by severe degenerative changes [9]. Within this realm, a great diversity of different types of injuries, which in turn produce a distinctive set of symptoms, currently exists. To begin, radiculopathy (also known as Sciatica) is a symptom rather than a diagnosis, and can be caused by several conditions, including herniated disc, degenerative disc disease, and spinal stenosis. Radiculopthy usually occurs when a disc or the nuclear material within it extends beyond its normal position and causes irritation to one or more nerve roots. This nerve root impingement may be the result of a direct impingement and/or an inflammatory cascade.

Differential diagnosis for discogenic pain can be difficult and according to Zhou [8], no unified diagnosis criteria exist for IDD at present. Discogenic pain is usually located in the low back area, with frequent radiation to bilateral lower extremities. Although exact criteria have not yet been agreed upon, fundamental and clinical differences exist between the various types of back pain due to disc injury. Of such, annular tears are the major forms of IDD, and include 3 types: circumferential tears, peripheral rim tears, and radial fissures. Circumferential tears may represent the effects of interlaminar shear stresses, possibly from compressive stress [9]. Peripheral rim tears are more frequent in the anterior annulus [16] and may be associated with boney outgrowth and/or trauma [9]. Radial fissures progress outwards from the nucleus, usually posteriorly or posteriolaterally [16], by cyclic loading and compression. Radial fissure are associated with nucleus degeneration, such that, when radial fissure allow gross migration of nucleus relative to annulus, to the extent that that disc periphery is affected, then the disc is described as herniated or prolapsed [9]. Depending on the extent of nucleus migration, the disc herniation may result in protrusion, extrusion or sequestration of nuclear material. If the nucleus pulposus protrudes into the annulus fibrosis, "true sciatica" may be the result of mechanical, chemical and/or inflammatory irritation to nerve roots.

Symptoms associated with a disc protrusion consist of pain, numbness and/or tingling in the low back that descends into the buttock, 
thigh, lower leg and/or foot, depends on the extent of irritation. A sequestration occurs when the degenerative disc allows a free fragment of nucleus pulposus and/annular material to form. A displaced sequestered fragment occurs when a free fragment dislodges in the spinal canal and foramen [17].

Although differential diagnosis may be a difficult task; it serves as a valuable screening tool for prescribing optimal therapeutic intervention (s). In terms of evaluating optimal therapeutic interventions, the current practices being used to treat degenerative IVD disorders may prove to be both costly and therapeutically ineffective. If non-surgical spinal decompression may aid in regenerating a selected group of IVD conditions, then perhaps this therapy may prove to be a more effective means of treating such conditions.

\section{Current practices with disc degeneration}

Lumbar spine surgery, due to conditions associated with Intervertbral Disc Disorders (IDD), is at its highest rate (by fivefold) in the US among developed countries [18]. These increasing surgical rates may have a tremendous effect on the health care market, and as a result, the socioeconomic burden of IDD is enormous. Within the orthopedic, neurologic and chiropractic literature, an intense debate over the effectiveness of surgical versus non-surgical techniques, currently exists. As a result of the vast array of contradictory reports, all but one conclusion is certain: an optimal treatment strategy remains unclear. The objective of this section of is to present the most recent studies investigating cost analysis techniques, socioeconomic evaluations, and the effectiveness and efficacy of surgical and non-surgical procedures.

The spine surgery rate in the US is the highest in the world $[19,20]$. With regards to lumbar fusion surgery, Deyo and Mirza [19] address the issue of increasing spine surgery rates and the trends in the various types of spine surgery procedures. The authors indicate that rate of spinal fusion surgery has increased $77 \%$ between 1996 and 2001 [19]. In spite of these significant increases, there is no epidemiologic data that demonstrates an increase in spinal disorders or a need for the increase in procedures [19]. This trend (s) may not be in the best interest of the patient; perhaps influenced by conflicts of interests in practiced patterns [20], such as the FDA's (define) approval of intervertebral fusion cages-at that time-and/or insurance company's willingness to reimburse.

Rapidly increasing surgical rates have a cautious effect on the health care market, and due to limited health care budgets, economic evaluations of the efficiency and effectiveness of spine surgical rates are becoming increasingly important [21]. Consequently, cost-effectiveness is becoming the primary evaluation criterion within healthcare [22]. The cost-effectiveness model takes into account the patient, the societal and the healthcare perspectives. The interpretation of both treatment costs and treatment effects may be difficult to determine, and become even more difficult across cultural and socioeconomic borders [18]. In fact, there are only a limited amount of studies estimating cost at the patient level and average estimates available (hospital charges) that currently exist [19].

In a report attempting to discuss the accuracy of measuring socioeconomic status with regards to lumbar disc disease, Katz [18] reviewed some of the various socioeconomic factors and their subsequent consequences. The United States Census records various socioeconomic factors, such as an individual's income, occupation and educational accomplishments; however, the methodology of collecting these various socioeconomic factors was coupled with challenges and inconsistencies [18]. One such inconsistency is cost analysis. Cost analysis can be subdivided into direct and indirect expenses. Direct costs include services, such as hospitalization, outpatient visits, medications, assistive devices, diagnostic test, and alternative therapies. Indirect costs involve productivity, and include the cost of lost wages due to work missed, reduced productivity, and an array of disability expenses $[8,20,23,24]$. Katz [18] suggests that the cost-effectiveness of instrumented fusion surgery is extremely high; therefore, should require thoughtful consideration of the value of fusion-type surgery.

In one cost-effectiveness evaluation, Fritzell et al., [23] examined the cost-effectiveness of lumbar fusion surgery for patients suffering with Chronic Low Back Pain (CLBP). In this study, Fritzell et al., [23] defined chronic pain as patients suffering with severe and therapy-resistant low back pain for at least two years. Data collection included outcomes from 284 patients, and randomization of patients occurred with patient receiving either surgical or nonsurgical treatment. This randomized clinical trial included four groups, of which three out of the four groups underwent a different type of surgical procedure. The different types of surgical procedures varied in invasiveness and complexity. A limitation of the Fritzell et al., [23] study was that the control group, which received unspecified physiotherapy, had already failed conservative therapy before this study. In addition, the authors failed to mention any specifics on the exact therapy applied to the non-surgical group during the study. Cost-effectiveness analysis was based on an assortment of direct and indirect variables, and the treatment's success was based on subjective measures. Fritzell et al., [23] state that societal cost, which is the sum of both the indirect and the direct cost variables, per patient in the surgical group was significantly higher as opposed to the non-surgical group. However, the cost-effectiveness of surgery was favorable because surgical patients' experienced significantly better results after the two-year follow up, than the non-surgical group. As a result, Fritzell et al., [23] concluded that the cost-effectiveness of spinal fusion depends on the decision-makers willingness to pay. The willingness to pay refers to the values (direct or indirect) that are most important to the individual and/or society. By introducing value as an outcome measure, the patients' own opinions of treatment are evaluated. The conclusion of the Fritzell et al., [23] study may reflect a dilemma in cost-effectiveness evaluation. This dilemma refers to evaluating the various aspects decision-makers willingness-to-pay, of which remains a controversial issue. Controversies arise due to the abundance of different outcome parameters and diversified study populations [22].

As a result, a short term cohort study [22], sought to develop a method of evaluating the cost-effectiveness of lumbar spinal fusion using an activity-based-costing analysis method. The authors investigate the determinants of cost-effectiveness at the individual patient-level. In addition, the authors compared the efficiency of anterior verses posterior instrumentation. This study [22] included a cohort of 136 patients with CLBP. All of these patients received a form of surgical treatment, and participated in a two-year postoperative follow-up survey. Prior to data collection, the authors expected a positive correlation between cost and effects related to the various types of lumbar fusion surgery [22].

Similar to the Fritzell et al., [23] study, the Soegaard et al., [22] study also involved surgical procedures that varied in invasiveness and complexity, and included non-instrumented and instrumented 
fusions. During the two-year follow-up period, a series of questionnaires monitored any subjective changes. A cost analysis at the patient-level, by means of activity-based costing, net-benefit approach and regression models, revealed determinants for cost and effects. Soegaard et al., [22] support that their net-benefit approach is a valuable mechanism for investigating the determinants of cost-effectiveness. Interestingly, contrary to their initial hypothesis, Soegaard et al., [22] suggests that no correlation exists between treatment cost and the various effect measures; yet the authors suggest that all groups were not only satisfied with the procedure, but also demonstrated significant clinical improvement. Poor lifestyle habits, such as smoking and/or psychosocial issues, can have a direct impact on net-benefit of lumbar fusion surgery [22]. In addition, the comparison the efficiency of anterior verses posterior instrumentation revealed inconclusive results. As a result of this study, Soegaard and colleagues [22] stress the need to further focus on determinants of cost-effectiveness, and that an individual patient's characteristics that are modifiable at a relatively low expense may have greater influence on cost-effectiveness than the surgical technique itself.

Studies focusing on an economic evaluation comparing operative and conservative (non-surgical) treatment are limited. In fact, it appears that the Fritzell et al., [23] study is the only study investigating this relationship. However, the limitation of the study, as stated above, may decrease the credibility of their findings. In response, an economic evaluation published by Rivero-Arias [25] attempts to determine the cost effectiveness of spinal fusion surgery when compared to intense rehabilitation programs. This study included 349 patients in total, of which 176 patients were randomized to spinal fusion surgery and 173 to intensive rehabilitation. The subjective outcome assessment was determined via an array of questionnaires, more specifically expressed as Quality-Adjusted-Life-Years (QALY). This data collection took place at 6,12 and 24 months post treatment. In addition, the cost of each subject's treatment was broken down and calculated based on a series of guidelines, which were thoroughly expressed in the report.

Rivero-Arias and colleagues [25] suggest that intensive rehabilitation is substantially less costly than surgery. In fact, two years after initial treatment, some patients' required additional treatment; of which, the cost difference still favored intensive rehabilitation. Rivero-Arias and colleagues [25] of this study suggest that spinal fusion surgery, as a first line treatment for chronic LBP, is not a cost effective health care option [25]. However, like the Fritzell et al., [23], the rehabilitation therapy program discussed is not clearly defined. In fact, the only detail mentioned of the program was some form of cognitive behavioral therapy.

In 2005, a prospective cohort study [26] was published, documenting 10-year outcomes of patients treated surgically or non-surgically. In this study, also called the maine spine study, the authors focused on an assortment of subjective factors, and selectively subdivided patients into either surgical or non-surgical groups. As a result, patients undergoing surgery had worse baseline symptoms and functional status than those initially treated non-surgically. Various follow-up questionnaires distributed at 3,6,12 months, and 10-years following initial treatment monitored subjective factors. Results illustrate that after a 10-year follow up for spinal stenosis treatment (both surgical and non-surgical), $50 \%$ of patients improved. Approximately $69 \%$ of those treated surgically for disc herniation, and $61 \%$ of those treated non-surgically, improved [26]. This data suggests that that half the stenosis patients and more than a third of the disc herniation patient found no improvement, and possibly a worsening of their conditions. However, the authors [26] interpret their results as suggesting that surgical patients reported better functional status and were more satisfied with the current state after ten years; yet, regardless of initial treatment, there was no significant difference with pain qualities and work/disability status with both groups. Although, the authors [26] conclude that their results support the relative benefit of surgery for properly selected candidates, the inconsistencies in this study are obvious.

Subjective outcomes used to evaluate the effectiveness of treatment seem to be the dominant factor in the majority of studies reviewed in this report. However, the mechanism of gathering the various subjective outcome features does not seem to be consistent across the board. The majority of the studies discussed in this report recognize that health-related quality of life is an important outcome $[18,24,25]$. By introducing value as an outcome measure, the patient's opinions of treatment are evaluated. In addition to the Rivero-Arias [25] study that utilized a QALY assessment, another study performed by Hansson \& Hansson, [24] used the same QALY assessment to investigate the cost and utility of lumbar disc herniation surgery. This cohort study [24] included 1,146 patients suffering with low back pain and/or radicular symptoms, of which each patient operated on was individually matched with one treated conservatively. Like the previous studies, the cost-effectiveness analysis is based on an assortment of direct and indirect variables. Pre-and-post treatment QALY surveys were analyzed and compared. The Hansson E et al., [24] report that medical costs are much higher for surgical treatments; however, the total cost (direct and indirect) is lower among those treated surgically. Thus, this study [24] supports surgery for carefully selected patients, and suggests that surgery for lumbar disc herniation is cost-effective.

Alternatively, van Tulder [21] suggest that the effectiveness of most spinal surgeries has yet to meet proper evidence-based guidelines. In addition, there is a lack of evidence, if any exists, that justifies the most updated technological procedures [8,21]. If there is inadequate evidence, then these procedures may be classified as experimental and investigational.

van Tulder [21] gathered data from Cochrane Database Review Group and COST B13 European Guidelines. These resources act as a collection agency, of which they collect and store up-to-date information on the effectiveness of medical interventions. van Tulder and colleagues The authors of this study [21] used the most recent literature published, to demonstrate the lack of strong evidence that exist in the field of invasive-surgical treatment modalities for spine-related conditions.

van Tulder and colleagues [21] subdivided their review into different categories, ranging from mild injection therapy to severe spinal surgery. Injection therapy may involve anaesthetics (including morphine) and/or steroid injections into various regions of the facet articulation, the lumbopelvic ligaments, the intervertebral disc and intramusclular trigger points. After reviewing the most current available data, the authors suggest that no sufficient evidence exists favoring the effectiveness of injection procedures, and thus they are not recommended [21].

Van Tulder [21] discuss surgical interventions and subdivide these procedures in to 1) methods attempting to address degenerative diseases and 2) methods attempting to address disc prolapse. Of all the 
various interventions mentioned in their review, surgical interventions seem to include the greatest quantity of studies; however, the studies included in this analysis appear to involve a lot of confounding and limiting factors, and thus, the authors [21] conclude that there is currently insufficient evidence to draw any conclusions except for that surgical discectomy may provide efficient clinical relief for carefully selected patients with sciatica due to lumbar disc prolapse that fail to resolve with conservative management.

Awad \& Moskovich, present a review documenting some of most current and most effective operative and non-operative methods applied to the treatment of lumbar disc herniation [27]. As per the non-operative treatment, the authors appear to predominately review the literature that refutes the effectiveness and/or reliability of these approaches; but yet, the authors agree that most patients can be treated successfully by non-operative means [27]. Of the vast array of options that exist, the authors favor physical therapy and/or lifestyle behavioral modifications.

Within the surgical treatment realm, the Awad and Moskovisch [27] refer to studies that evaluate the long term vs. short-term success of the vast array of surgical treatment options. The results of this review, suggests that no one method of non-operative or operative treatment seems to be more superior to another [27]. In fact, the best outcomes can only result from an evidence-based approach that is tailored to the individual patient's spinal pathology, socioeconomic status and behavioral environment.

As per minimally invasive and/or non-operative treatment options, Zhou and Abdi [8] suggest that these types of treatments provide an "alternative" with appeal of cost-effectiveness and possibly less long-term side effects. However, according to Zhou and Abdi [8], the value of these treatments, which can include anti-inflammatory drugs, physiotherapy, rehabilitation, antidepressants, antiepileptics, and acupuncture are yet to be established [8].

As diverse as the above studies may appear, there are some similarities that most of them share. First, most of the above studies do not define conservative treatment. Secondly, the majority of all of the above studies stressed the importance of candidate selection. Finally, with exception to the Maine Spine Study, most of the above studies 16-19 used two-year follow up subjective outcome measures. Unfortunately, in terms of cost effectiveness, if an increasing number of patients pursue other options beyond two years, then the conclusion of these studies may be subject to alteration.

The debate over the effectiveness of surgical versus non-surgical techniques that currently exists reveals inconclusive results. As demonstrated above, much of the literature suggests that there is no definitive therapy for treating conditions associated with IDD. In fact, all versions of treatment options demonstrate a wide range of different levels of success and/or offer little available evidence. In turn, surgical procedures appear to be just as successful as they are unsuccessful. In fact, even the so-called "improvements" may have been relatively insignificant.

This review on the current practices emphasizes the need for better treatments for conditions associated with IDD. The issue of socioeconomic evaluations, and the effectiveness and efficacy of surgical versus non-surgical procedures, needs to be a foremost concern of health care leaders.

\section{Non-Surgical spinal decompression}

The IVD is composed of an outer layer of organized collagen fibrils and an inner nucleus pulposus; this nucleus prominently consists of water [2,3]. As mentioned above, regardless of the cause of IVD injury, a gradual loss of proteoglycans and water initiates the degenerative process [12-14].

The water content of the nucleus pulposus can be a reliable measure of its degenerative status. This degeneration status can be visualized and measured using specific Magnetic Resonance Imaging (MRI) signals [8]. A case study investigating nutritional supplementation to treat symptomatic disc degeneration demonstrates this rehydration phenomenon [8]. After a two-year period of nutritional supplementation with glucosamine and chondroitin sulfate, pre-and-post MRI's of the lumbar spine demonstrated signal intensity changes. The researchers reported improvement of the structural quality of the disc cartilage, which is associated with increased water content, and visualized by brightening of the T2-weighted MRI signal. This evidence supports the notion that a degenerative IVD has the ability to regenerate, repair and rehydrate.

If a degenerative disc can regenerate and repair, then its water content may determine regeneration and repair capabilities. By exploring the nature of the IVD and the issues on healing, a resulting theory applies. If an IVD can degenerate under specific conditions, then the reverse may also be true; therefore, degenerative spinal conditions may have the ability to regenerate with specific therapeutic procedures.

A herniated IVD is under constant elevated intradiscal pressure $[2,28,29]$ and if intradiscal pressure can reduce, then perhaps a healing/regenerative response can occur. Non-surgical technology can therapeutically reduce intradiscal pressure and may allow for degenerative spinal conditions to regenerate, and more specifically, rehydrate. Of these advanced non-surgical techniques, spinal decompression appears to be the front-runner in correction of herniated disc disorders. Non-surgical spinal decompression has the ability to reduce intradiscal pressure and specifically target the problem area of the disc [1,2,14,29-31]. As a result, non-surgical spinal decompression can improve the objective findings in patients suffering with symptoms caused by IVD irritation. The studies summarized below document these objective physiologic benefits. The objective modalities include pre-and-post changes in Computed Tomography (CT) images, Magnetic Resonance Imaging (MRI) and various nerve conduction changes.

Tilaro and Miskovich [32] conducted a nonrandomized retrospective analysis to determine if Vertebral Axial Decompression (VAX-D) therapy could externally decompress irritated nerve roots and improve sensory nerve dysfunction. Their study focused on an objective means of assessing the dysfunction of peripheral sensory nerves. The VAX-D therapeutic table theoretically decompresses nerve roots by reducing intradiscal pressure, by providing a monitored distractive force to the lumbar spine [32]. This distractive/decompressive force can reduce intradiscal pressure, which ultimately enables retraction of the herniation. Subjects of this study suffered with chronic low back pain and radiculopathy. In addition, they also demonstrated abnormal sensory nerve function. An electronic nerve conduction devise, known as the Current Perception Threshold (CPT) neurometer determined the degree of sensory nerve dysfunction in all subject. The CPT neurometer can measure transcutaneous electric nerve stimulation 
and determine the efficiency and/or functioning of various pre-determined nerve root fibers. In addition to this CPT device, all subjects demonstrated a positive orthopedic test (Straight Leg Raise) and positive imaging studies before the therapy began. Investigators obtained CPT evaluations of all subjects before and after a series of VAX-D treatment sessions.

According to pre-and-post CPT neurometer measurements, Tilaro and colleagues suggest that ninety-one (91\%) of impaired sensory nerves demonstrated improved neurological function [32]. Of these, $64 \%$ of those impaired nerves returned to normal function, $27 \%$ improved but did achieve absolute normal function, and $9 \%$ either demonstrated no improvement and/or worsened. These results suggest the VAX-D therapeutic table is capable of affecting spinal sensory nerve dysfunction in abnormal nerves, secondary to a compressive radiculopathy.

Using this same technology, Dyer presented multiple case studies that demonstrated pre-and-post Magnetic Resonance Imaging (MRI) changes [33]. Dryer mentions that "retraction of protruding segments has been observed in other cases and is an interesting phenomenon that intrigues the skeptics; however it has not been a consistent finding in all cases that have achieved complete remission" [24]. Therefore, the significance and correlation has yet to be established.

In another study, conducted at St. Louis University School of Medicine, Eyerman presented MRI evidence of non-surgical repair of herniated lumbar disc [2]. Using a spinal decompression table, called the Decompression Reduction and Stabilization system (DRS), Eyerman demonstrates that non-invasive spinal decompression could improve the objective findings in patients suffering with low back pain and radiculopathy. In this study, electromyography and pre-and-post Magnetic Resonance Imaging (MRI) findings demonstrate objective changes. Eyerman explains that with discography, a herniated disc is under constant elevated intradiscal pressures [2]. Other studies, including a study at the Department of Neurosurgery in Rio Grande Regional Hospital, indicate that it is possible to lower pressure in the nucleus propulus of herniated lumbar disc when a distraction tension is applied [34]. Therefore, spinal decompression therapy theoretically decompresses an over-pressurized disc.

Eyerman's study [2] involved patients suffering with lumbar radiculopathy and demonstrating a herniated disc (s) on MRI and abnormal nerve conduction findings on electromyophy. Electromyophy is similar to the CPT devise, such that it is an objective way to determine the status of irritated nerves. These objected modalities were performed within four weeks before and after treatment. Nerve root irritation was confirmed with electromyography, and the presence/ severity of a herniated disc was determined via MRI studies. In this study, Eyerman [2] clearly defines the treatment protocols and documents any subjective, mobility, and/or neurological exam changes throughout the treatment process.

According to pre-and-post MRI findings, Eyerman [2] suggest that disc herniations reduced significantly in 10 of the 14 subjects. Of these, six patients demonstrated $50-100 \%$ reductions, and four patients demonstrated $25-50 \%$ reductions. One patient, not only demonstrated reduction on post-MRI, but also demonstrated rehydration. Rehydration is evident by increase signal intensity on T2 weighted MRI scans. Eyerman [2] did not mention the evaluation criteria they used to measure the reductions or changes in pre-and-post MRI. However, there is promising information, which clearly demonstrates that, a degenerative and/or herniated disc have the ability to regenerate and rehydrate. This concept supports the above hypothesis.

Similarly, Onel and colleagues demonstrate the effects of a distractive force on a disc herniation using CT imaging [31]. To demonstrate these objective changes, the investigators of this study designed a traction table to fit into a CT scanner. Investigators obtained images of all subjects before and during the traction forces, as well as after a series of treatment sessions. A pre-CT scan and clinically significant signs and symptoms confirmed the subjects' diagnoses. Pre-and-post CT images reveal retraction of herniated nuclear material in 21 of the 30 patients. More specifically, after a series of treatments, herniated nuclear material retracted in $78 \%$ of the medial disc herniations, $66 \%$ of the posterior-lateral herniations and $57 \%$ of the lateral herniations. Similar to the circumstances above, Onel and colleagues [31] theorize that a significant distractive force can cause a negative pressure gradient; thus allowing the disc to recede.

There are some similarities between the above studies. First, although subjective measures are not the focus of any of these studies, all of the authors mention a drastic improvement in symptomatology, which include an improvement in subjective complaints and other orthopedic/neurological findings. Secondly, all of the treatment procedures require the patient to be a supine position, with both knees in semiflexion. Additionally, each treatment protocol consisted of 20-25 sessions over 4-6 week duration, and the specific therapy involved both loading and unloading forces. Loading and unloading cycles are consecutive alternating compression and traction movements [1].

The above studies demonstrate that by applying a significant decompressive and/or distractive force to a herniated disc, an objective change can occur. The mechanisms responsible for these changes are associated with a reduction of intradiscal pressure. Basic osmotic changes can cause this reduction of intradiscal pressure. Repetitive traction followed by compression of the disc produce "imbibation and osmosis" of water into the disc rehydrating or regenerating the disc height [1]. Some of this research demonstrates that by introducing loading and unloading cycles in the spine, we can literally "suck" water back into the disc and rehydrate it. However, it appears, as though there have only been a few [2,3] isolated cases that demonstrate true rehydration of disc. Most of the literature demonstrates reduction, regression, retraction and/or disappearance of herniation.

\section{Conclusion}

The concepts presented in each section of this report were supplied with the objective of introducing a notion on the ability of degenerate Intervertebral Disc (IVD) disorders to regenerate or rehydrate. This paper attempted to explain this notion by exploring several different issues, such as anatomy and physiology, pathology and epidemiology, differential diagnosis, the current therapeutic practices being used to treat degenerative disc conditions, and a rationalization on how non-surgical spinal decompression may be able to regenerate a group of selective degenerative spinal conditions. With further research, this concept may lead to a paradigm shift in the pain management realm. Thus, patients can chose to receive corrective, non-invasive, close to zero risk therapy, rather than surgical/invasive interventions. 


\section{References}

1. Lerner B, Dideminico F (2005) The New Science of Spinal Regeneration. Mercola, California, USA.

2. Eyerman E (1998) MRI Evidence of Nonsurgical, Mechanical Reduction, Rehydration and Repair of the Herniated Lumbar Disc. Journal of Neuroimaging 8: 1-8.

3. van den Bussche H, Jahncke-Latteck AD, Ernst A, Tetzlaff B, Wiese B, et al. (2013) Satisfied general practitioners and critical nursing staff problems of interprofessional cooperation in the home care of dementia patients. Gesundheitswesen 75: 328-333.

4. Macario A, Pergolizzi JV (2006) Systematic literature review of spinal decompression via motorized traction for chronic discogenic low back pain. Pain Pract 6: 171-178.

5. Belavy DL, Quittner M, Ling Y, Connell D, Rantalainen T (2017) Cervical and thoracic intervertebral disc hydration increases with recumbency: a study in 101 healthy volunteers. Spine J.

6. Hwang D, Kim S, Abeydeera NA, Statum S, Masuda K, et al. (2016) Quantitative magnetic resonance imaging of the lumbar intervertebral discs. Quant Imaging Med Surg 6: 744-755.

7. Peng B, Hao J, Hou S, Wu W, Jiang D, et al. (2006) Possible pathogenesis of painful intervertebral disc degeneration. Spine (Phila Pa 1976) 31 $560-566$.

8. Zhou Y, Abdi S (2006) Diagnosis and Minimally Invasive Treatment of Lumbar Discogenic Pain ??? A Review of the Literature. Clin J Pain 22: 468-481.

9. Adams MA, Roughley PJ (2006) What is intervertebral disc degeneration, and what causes it? Spine (Phila Pa 1976) 31: 2151-2161.

10. Paesold G, Nerlich AG, Boos N (2007) Biological treatment strategies for disc degeneration: potentials and shortcomings. Eur Spine J 16: $447-$ 468 .

11. Malko JA, Hutton WC, Fajman WA (1999) An in vivo magnetic resonance imaging study of changes in the volume (and fluid content) of the lumbar intervertebral discs during a simulated diurnal load cycle. Spine (Phila Pa 1976) 24: 1015-1022.

12. Sakai D, Mochida J, Iwashina T, Watanabe T, Nakai T, et al. (2005) Differentiation of mesenchymal stem cells transplanted to a rabbit degenerative disc model: potential and limitations for stem cell therapy in disc regeneration. Spine (Phila Pa 1976) 30: 2379-2387.

13. Sakai D, Mochida J, Iwashina T, Hiyama A, Omi H, et al. (2006) Regenerative effects of transplanting mesenchymal stem cells embedded in atelocollagen to the degenerated intervertebral disc. Biomaterials 27: $335-345$.

14. Cheug KMC, Ho G, Chan D (2004) Intervertebral Disc Regeneration by Use of Autologous Mesenchymal Stem Cells, an Experimental Model in Rabbits 2004 Abstracts from the Scoliosis Research Society.

15. Crock HV (1970) A reappraisal of intervertebral disc lesions. Med J Aust. 1: 983-989.

16. Osti OL, Vernon-Roberts B, Moore R, Fraser RD (1992) Annular tears and disc degeneration in the lumbar spine. A post-mortem study of 135 discs. J Bone Joint Surg Br 74: 678-682.

17. Cox JM (1990) Low Back Pain: mechanism, diagnosis, and treatment. Williams \& Wilkins, Baltimore, USA.
18. Katz JN (2006) Lumbar disc disorders and low-back pain: socioeconomic factors and consequences. J Bone Joint Surg Am 88: 21-24.

19. Deyo RA, Mirza SK (2006) Trends and variations in the use of spine surgery. Clin Orthop Relat Res 443: 139-146.

20. Cook RW, Weiner JA, Schallmo MS, Chun DS, Barth KA, Singh SK, et al. (2017) Effects of Conflicts of Interest on Practice Patterns and Complication Rates in Spine Surgery. Spine (Phila Pa 1976) 42: 13221329.

21. van Tulder MW, Koes B, Seitsalo S, Malmivaara A. Outcome of invasive treatment modalities on back pain and sciatica: an evidence-based review. Eur Spine J 15: 82-92.

22. Soegaard R, Christensen FB, Christiansen T, Bunger C (2007) Costs and effects in lumbar spinal fusion. A follow-up study in 136 consecutive patients with chronic low back pain. Eur Spine J 16: 657-668.

23. Fritzell P, Hagg O, Jonsson D, Nordwall A, Swedish Lumbar Spine Study Group (2004) Cost-effectiveness of lumbar fusion and nonsurgical treatment for chronic low back pain in the Swedish Lumbar Spine Study: a multicenter, randomized, controlled trial from the Swedish Lumbar Spine Study Group. Spine (Phila Pa 1976) 29: 421-434.

24. Hansson E, Hansson T (2007) The cost-utility of lumbar disc herniation surgery. Eur Spine J 16: 329-337.

25. Rivero-Arias O, Campbell H, Gray A, Fairbank J, Frost H, et al. (2005) Surgical stabilisation of the spine compared with a programme of intensive rehabilitation for the management of patients with chronic low back pain: cost utility analysis based on a randomised controlled trial. BMJ 330: 1239.

26. Atlas SJ, Keller RB, Wu YA, Deyo RA, Singer DE (2005) Long-term outcomes of surgical and nonsurgical management of sciatica secondary to a lumbar disc herniation: 10 year results from the maine lumbar spine study. Spine (Phila Pa 1976) 30: 927-935.

27. Awad JN, Moskovich R (2006) Lumbar disc herniations: surgical versus nonsurgical treatment. Clin Orthop Relat Res 443: 183-197.

28. Ramos G, Martin W (1994) Effects of vertebral axial decompression on intradiscal pressure. J Neurosurg. 81: 350-353.

29. Daniel DM (2007) Non-surgical spinal decompression therapy: does the scientific literature support efficacy claims made in the advertising media? Chiropr Osteopat 15: 7.

30. Liang HF, Liu SH, Chen ZX, Fei QM (2017) Decompression plus fusion versus decompression alone for degenerative lumbar spondylolisthesis: a systematic review and meta-analysis. Eur Spine J.

31. Onel D, Tuzlaci M, Sari H, Demir K (1989) Computed tomographic investigation of the effect of traction on lumbar disc herniations. Spine (Phila Pa 1976) 14: 82-90.

32. Tilaro F, Miskovich F (1999) The Effects of Vertebral Axial Decompression On Sensory Nerve Dysfunction In Patients with Low Back Pain And Radiculopathy. Canadian Journal of Clinical Medicine 6.

33. Dyer AE (1999) The Science of Decompression. The American Association of Orthopedic Medicine, Illinois, USA.

34. O'Hara K (2004) Decompression: The treatment for Back Pain. Occupational Medicine 11. 